Ann. Génét. Sél. anim., I980, 12 (3), 24I-254.

\title{
Gene cou nu, poids corporel et paramètres anatomiques et physiologiques des poulettes et poules adultes selon la température
}

\author{
L. E. MONNET $\left(^{*}\right)$, A. BORDAS et P. MÉRAT \\ avec la collaboration technique de M. DUMORTIER \\ Laboratoire de Génétique Factorielle \\ I.N.R.A., \\ 78350 Jouy-en-Josas
}

\begin{abstract}
Résumé
Des poules des trois génotypes $\mathrm{NaNa}$ (homozygotes "Cou nu "), Nana+ (hétérozygotes "Cou nu ") et $n a+n a+$ (plumage normal), issues des mêmes parents, ont été distribuées dans deux lots : "témoin " (gardé à une température modérée, avec des fluctuations suivant les variations climatiques) et "chauffé " ( $3 \mathrm{I}^{\circ} \mathrm{C}$ constamment de 4 à 39 semaines d'âge). Dans le lot témoin, alors que les poids des trois génotypes à ro semaines d'âge différaient peu, les gains de poids après cet âge étaient très inférieurs pour le génotype $N a N a$ comparé à $n a^{+} n a^{+}$, conduisant à des poids corporels à $\mathrm{I} 8,27$ et 39 semainies d'âge nettement moins élevés pour les homozygotes "Cou nu "; 1a longueur des tarses, corrélativement, étant légèrement inférieure. La taille des barbillons était, par contre, supérieure pour les femelles $N a N a$. Leur température rectale et la température superficielle des tarses étaient légèrement plus basses que chez les oiseaux à plumage normal. lue génotype hétérozygote était d'une manière générale intermédiaire, mais plus proche de l'homozygote $\mathrm{NaNa}$ pour la longueur des barbillons, la longueur et la température des tarses, et plus voisin du génotype $n a+n a+$ pour la température rectale.

En lot chauffé, à ro semaines d'âge et encore à 18 semaines, les femelles "Cou nu " homozygotes sont les plus lourdes et les hétérozygotes intermédiaires, mais la différence s'atténue avec l'âge et n'est plus significative à partir de 27 semaines. Comme en lot témoin, les poules "Cou nu " ont des barbillons de plus grande taille. Leur température rectale et superficielle est aussi légèrement inférieure, mais ici la différence n'est pas significative. Eñfin, dans le lot à température élevée les poules à plumage normal ont un taux d'hématocrite plus bas.

Le poids corporel à 18 semaines, le poids adulte, la température du tarse et l'hématocrite présentent une interaction génotype $\times$ traitement significative.

Plusieurs variables physiologiques ou concernant la composition corporelle ont été mesurées seulement en lot chauffé. Parmi elles, le rapport de l'eau à l'aliment ingéré est plus faible chez les poules "Cou nu " que chez les autres, alors que le poids de matière sèche dans les déjections est plus élevé, en corrélation avec l'ingestion plus grande d'aliment. Le poids du plumage en p. roo du poids corporel augmente en passant đu génotype $\mathrm{NaNa}$ au génotype $\mathrm{Nana}{ }^{+}$, puis $n \mathrm{a}^{+} n \mathrm{a}^{+}$ conformément au résultat tsouvé antérieurement à Io semaines d'âge. Les poules "Cou nu " sont plus grasses, leurs thyroïdes sont de plus grande taille et leurs surrénales plus petites.
\end{abstract}




\section{Introduction}

Le gène "Cou nu " $(\mathrm{Na})$ se caractérise par une réduction des zones emplumées ou ptérylies (Hutr, I949). On peut supposer que ceci a des répercussions sur la régulation thermique. En réponse à un stress thermique, SmiTr et LEE (I977) obtiennent moins de mortalité pour des poussins hétérozygotes $\mathrm{Nana}^{+}$que pour des homozygotes $n a^{+} n a^{+}$.

Dans un article séparé (BORDAS et al., I980) est présentée une comparaison de la ponte, des caractéristiques des oufs et de l'efficacité alimentaire de poules des trois génotypes $\mathrm{NaNa}, \mathrm{Nana}^{+}$et $n a^{+} n a^{+}$, élevées à deux températures ambiantes différentes, modérée ou chaude. Cet article fait lui-même suite à une comparaison entre les mêmes génotypes, concernant la phase de croissance, jusqu'à Io semaines d'âge (MoNNE'r et al., I979). Il s'agit, à notre connaissance, des premiers travaux publiés concernant l'effet associé au gène "Cou nu " sur des performances quantitatives suivant la température ambiante. Le présent article complète ces résultats par la présentation, sur les mêmes animaux que dans le premier article cité, de données relatives au poids corporel des poulettes et des poules adultes et à divers paramètres anatomiques et physiologiques.

\section{Matériel et méthodes}

\section{I. - Materiel animal et conditions expérimentales}

Ils ont déjà été décrits en détail dans les précédents articles (Monnet et al., I979; Bordas et al., I 980). Nous n'en rappellerons donc ici que les points essentiels pour le présent travail.

Les poussins expérimentaux sont issus de parents tous deux hétérozygotes $\mathrm{Nana}^{+}$. Une éclosion (26.9.78) a fourni le lot "témoin ", une seconde, trois semaines plus tard, le lot "chauffé ". Chaque lot a été élevé au sol dans un local distinct, jusqu'à l'âge de I 8 semaines ( 55 o gardées par génotype et par lot). A cet âge, 40 poulettes par génotype, choisies au hasard, ont été mises en cages individuelles dans un bâtiment différent pour chaque lot. Les deux lots ont reçu jusqu'à la fin de l'expérience le même éclairage ( $14 \mathrm{~h}$ de lumière sur 24 heures) et le même aliment ad libitum (à $\mathrm{I} 6 \mathrm{p}$. Ioo de protéines totales et $2600 \mathrm{Kcal}$ E.M. $/ \mathrm{kg}$ à partir de I8 semaines).

A part la date d'éclosion et quelques différences mineures (BORDAS et al., I980), les deux lots se sont distingués essentiellement par la température ambiante réalisée. Pour le lot témoin, le chauffage a été arrêté à 4 semaines d'âge. Ultérieurement, le local était en partie soumis aux fluctuations climatiques, un chauffage d'appoint empêchant seulement que la température ne descende en-dessous de Io ${ }^{\circ} \mathrm{C}$. Pour le lot chauffé, la température était maintenue au voisinage de $3{ }^{\circ}{ }^{\circ} \mathrm{C}$ depuis l'âge de deux semaines jusqu'à la fin de l'expérience, avec des fluctuations dont $1^{\prime}$ amplitude journalière était toujours inférieure à $4^{\circ} \mathrm{C}$. Le tableau I du précédent article (BORDAS et al., I980) indique les températures extrêmes rencontrées entre 4 et Io semaines d'âge, entre Io et 27 semaines et de 27 à 39 semaines. Nous en retiendrons que, pour le lot témoin, la température moyenne se situait respectivement au voisinage de $20^{\circ} \mathrm{C}$, II ${ }^{\circ} \mathrm{C}$ et $\mathrm{I}^{\circ}{ }^{\circ} \mathrm{C}$ dans chacune des trois périodes 
mentionnées ci-dessus. Ainsi, une différence considérable de température a toujours été maintenue entre les deux lots; d'autre part, le lot témoin a subi un net refroidissement entre ro et 27 semaines d'âge.

Le degré hygrométrique n'a pas été relevé avec précision. Il se situait généralement en-dessous de $50 \mathrm{p}$. roo.

\section{2. - Critères mesurés}

Croissance des poulettes et poids adulte

Les pesées servant de repères pour la croissance pondérale après l'âge de Io semaines se sont situées à I 8 semaines (âge de passage en cages), 27 et 39 semaines. L'analyse de variance est présentée seulement pour le poids à $\mathrm{I} 8$ semaines et le poids moyen dans la période comprise entre 27 et 39 semaines.

\section{Mesures morphologiques}

I1 s'agit de : la longueur des barbillons $(\mathrm{cm})$; la longueur des tarses (cm); le diamètre des tarses (cm).

Ces mesures ont été effectuées au millimètre près pour les deux premières, au $\mathrm{I} / \mathrm{IO}^{\mathrm{e}}$ de millimètre pour la dernière, sur animaux vivants à 39 semaines d'âge.

Les paramètres physiologiques mesurés dans les deux lots sont :

- la température rectale (une mesure à 39 semaines d'âge par thermocouple à sonde, entre 9 et Io h du matin);

- la température du tarse ( $\mathrm{m}$ mesure par thermocouple à la surface du tarse à 33 semaines d'âge entre $\mathrm{I} 4 \mathrm{~h}$ et $\mathrm{I} 5 \mathrm{~h}$ );

- l'hématocrite (p. IOo) : une mesure sur prélèvement de sang à la veine alaire à 40 semaines d'âge.

Variables physiologiques et anatomiques mesurées seulement pour le lot chauffé Ces variables sont les suivantes :

- eau ingérée : consommation moyenne quotidienne, obtenue à partir de mesures journalières faites pendant 7 jours consécutifs ( $33^{\mathrm{e}}$ semaine d'âge).

Deux rapports en sont dérivés, celui de l'eau ingérée au poids corporel et de l'eau à l'aliment ingéré dans le même temps;

- p. Ioo d'eau des déjections : sur déjections recueillies individuellement pendant 48 heures, puis séchées 48 heures à l'étuve à $60^{\circ} \mathrm{C}$;

- poids de matière sèche des déjections (g).

- En fin d'expérience, au cours de la $4 \mathrm{I}^{\mathrm{e}}$ semaine d'âge, II poulettes ont été abattues dans chaque génotype et les variables anatomiques suivantes déterminées, en $\mathrm{p}$. Ioo du poids corporel :

- poids du plumage; 
- poids de graisse abdominale, incluant la graisse contenue dans la cavité abdominale (à l'exclusion de celle attachée à l'intestin), plus celle entourant le gésier (cf. RICARD et ROUviER, I967; RICARD, r974).

- poids des thyroïdes

- poids des surrénales

Le plumage et la graisse abdominale ont été pesés au gramme, les thyroïdes et surrénales au $\mathrm{r} / \mathrm{IO}^{\mathrm{e}} \mathrm{de} \mathrm{mg}$ près.

\section{3. - Analyse statistique des données}

Chaque caractère a fait l'objet à l'intérieur de chaque lot d'une analyse de variance à deux facteurs, génotype et famille de père. D'autre part, une analyse de variance à trois voies, génotype, famille de père et traitement, a été pratiquée sur l'ensemble des deux lots.

Lorsque l'analyse de variance indiquait un effet significatif du génotype, les génotypes ont été comparés deux à deux par la méthode du $t$ corrigé.

\section{Résultats}

Pour le lot témoin, les tableaux I et 2 donnent respectivement les valeurs moyennes par génotype et l'analyse de variance à deux facteurs (génotype famille) concernant les mesures de croissance des poulettes, du poids adulte, les variables morphologiques et physiologiques mesurées sur l'ensemble des animaux. Des tableaux analogues (3 et 4) se rapportent au lot chauffé.

Pour ces mêmes variables, le tableau 5 donne les valeurs moyennes par lot, tous génotypes réunis, et le tableau 6 indique la signification des analyses de variance à trois facteurs (lot - génotype - famille).

Dans le tableau 7 sont présentées les valeurs moyennes par génotype des variables physiologiques et anatomiques mesurées seulement en lot chauffé; le tableau 8 contient les analyses de variance correspondantes (facteurs génotypefamille) limitées au premier groupe des variables, étant donné le petit nombre de poules sur lesquelles ont été mesurés les variables anatomiques.

\section{I. - Croissance des poulettes en lot témoin et en lot chauffé}

En lot témoin, (tab1. I et 2), nos résultats font apparaître une nette inflexion de la courbe de croissance à partir de I 8 semaines, et une croissance pondérale très faible au-delà de 27 semaines. Ils mettent également en évidence des différences suivant les génotypes, l'individu sauvage manifestant, en moyenne, les plus forts gains de poids. A l'âge de 18 semaines, L'individu normalement emplumé est de 7 p. roo plus lourd que la poulette homozygote "cou nu "; l'hétérozygote est intermédiaire, relativement proche du mutant. A 27 semaines, la différence de poids entre les deux homozygotes atteint I2 p. roo; l'hétérozygote est de 7 p. Ioo inférieur à l'individu sauvage, et significativement différent des deux homozygotes. Ces différences restent respectivement de II et $6 \mathrm{p}$. Ioo au stade adulte (39 semaines). 


\section{TABLEAU I}

Croissance des poulettes, poids adulte, variables morphologiques et physiologiques: valeurs moyennes par génotype, lot témoin

Growth of females, adult body weight, morphological and physiological variables: mean values according to genotype, control group

\begin{tabular}{|c|c|c|c|}
\hline \multirow{2}{*}{ Paramètres } & \multicolumn{3}{|c|}{ Génotypes } \\
\hline & $\mathrm{NaNa}$ & Nana + & $n a+n a+$ \\
\hline Poids à i o s., rappel (g) & I 246 & I 188 & I 230 \\
\hline Poids à 18 s. (g) & $2357^{a}$ & $2395^{a}$ & $2569^{b}$ \\
\hline Poids à 27 s. (g) . . . . . & $25^{8} 3^{a}$ & $2719^{b}$ & $2914^{c}$ \\
\hline Poids à 39 s. $(\mathrm{g})$. . . . . & $2615^{a}$ & $2740^{b}$ & $2896^{c}$ \\
\hline Poids moyen entre 27 et $39 \mathrm{~s}$. & $2599^{a}$ & $2729^{b}$ & $2905^{c}$ \\
\hline Longueur des barbillons (cm) & $4,32^{a}$ & $4, \mathrm{I} 5^{a}$ & $3,72^{b}$ \\
\hline Longueur des tarses $\quad(\mathrm{cm})$ & I I, $08^{a}$ & I I, $05^{a}$ & II, $3 I^{b}$ \\
\hline Diamètre des tarses & I, I I & $\mathbf{I}, \mathrm{OI}$ & 1,02 \\
\hline Température rectale & $40,22^{a}$ & $4^{0}, 3^{8 b}$ & $40,39^{b}$ \\
\hline Température du tarse $\left({ }^{\circ} \mathrm{C}\right)$. & $32,93^{a}$ & $32,92^{a}$ & $34,07^{b}$ \\
\hline Hématocrite $\quad(\%)$ & $3 I, 7$ & 31,2 & $3 \mathrm{I}, 5$ \\
\hline
\end{tabular}

Ires moyennes suivies de lettres différentes sont significativement différentes.

TABLEAU 2

Croissance des poulettes, poids adulte, variables morphologiques et physiologiques Lot témoin - Analyse de variance

Growth of females, adult body weight, morphological and physiological variables Variance analysis - control group

\begin{tabular}{|c|c|c|c|c|}
\hline \multirow{2}{*}{ Paramètres } & \multicolumn{4}{|c|}{ Variance et signification } \\
\hline & $\begin{array}{c}\text { Génotype } \\
(2)\end{array}$ & $\underset{(8)}{\text { Famille }}$ & $\begin{array}{l}\text { Interaction } \\
\text { (I6) }\end{array}$ & $\begin{array}{c}\text { Résiduelle } \\
(83)\end{array}$ \\
\hline Poids à I 8 s. . . . . . . . . & $576400(* * *)$ & I $54206(* * *)$ & 40,8 I 8 & $3 \mathrm{I} 42 \mathrm{I}$ \\
\hline Poids moyen entre 27 et $39 \mathrm{~s}$. & $963945(* * *)$ & $400580(* * *)$ & $67 \times 56$ & 70334 \\
\hline Longueur des barbillons . . & $3,70(* * *)$ & $\mathrm{I}, \mathrm{O} 5(* * *)$ & 0,17 & 0,22 \\
\hline Longueur des tarses . . . . & $0,8 \mathrm{I}(* *)$ & $0,93\left(^{* *}\right)$ & 0,15 & 0,16 \\
\hline Diamètre des tarses $(\times$ Ioo $)$ & 26,1 & $124,7(* * *)$ & 23,4 & 21,8 \\
\hline Température rectale. . . . & $0,242(* *)$ & 0,107 & 0,086 & $0,06 \mathrm{I}$ \\
\hline Température du tarse . . . & I $3,30(* * *)$ & $7,42(* * *)$ & 0,96 & 1,46 \\
\hline Hématocrite. . . . . . . & $2, \mathrm{I}$ & I $9,0(*)$ & 3,4 & 7,2 \\
\hline
\end{tabular}

- Entre parenthèses : d.1.

$-\left(^{*}\right)\left({ }^{* *}\right)(* * *)=$ significatif respectivement au seuil 5 , I et 0, I p. Ioo. 
TABLEAU 3

Croissance des poulettes, poids adulte, variables morphologiques et physiologiques Valeurs moyennes par génotype - Lot chauffé

Growth of femals, adult body weight, morphological and physiological variables: mean values according to genotype - heated group

\begin{tabular}{|c|c|c|c|}
\hline \multirow{2}{*}{ Paramètres } & \multicolumn{3}{|c|}{ Génotypes } \\
\hline & $N a N a$ & $\mathrm{Nana}^{+}$ & $n a^{++}+n a$ \\
\hline 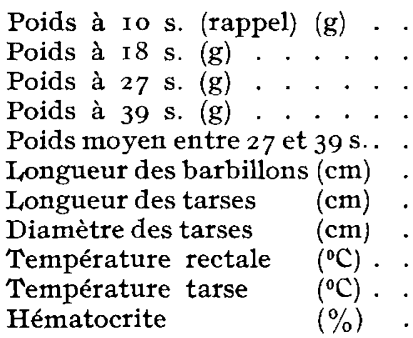 & $\begin{array}{ll}\text { I I I } 6 \\
23^{6} 3^{a} \\
2632 \\
274^{8} \\
2665 \\
4,37^{a} \\
\text { I I, } 23 \\
\text { I, } 03 \\
40,39 \\
35,79 \\
30,7^{a}\end{array}$ & $\begin{array}{l}\text { I } 083 \\
23 \text { I } 3^{a b} \\
2596 \\
267 \text { I } \\
2611 \\
4,02^{b} \\
11,31 \\
1,05 \\
40,53 \\
35,7 \text { I } \\
30,6^{a}\end{array}$ & $\begin{array}{rl} & 992 \\
2 & 2 \mathrm{I} \mathrm{I}^{b} \\
2 & 499 \\
2 & 560 \\
2 & 525 \\
3,82^{b} \\
\text { I I, }{ }^{\mathrm{I}} \\
\mathrm{I}, \mathrm{O} 4 \\
40,6 \mathrm{I} \\
35,86 \\
28,7^{b}\end{array}$ \\
\hline
\end{tabular}

Les moyennes suivies de lettres différentes sont significativement différentes.

TABLEAU 4

Croissance des poulettes, poids adulte, variables morphologiques et physiologiques Lot chauffé - Analyses de variance

Growth of femals, adult body weight, morphological and physiological variables: Variance analysis, heated group

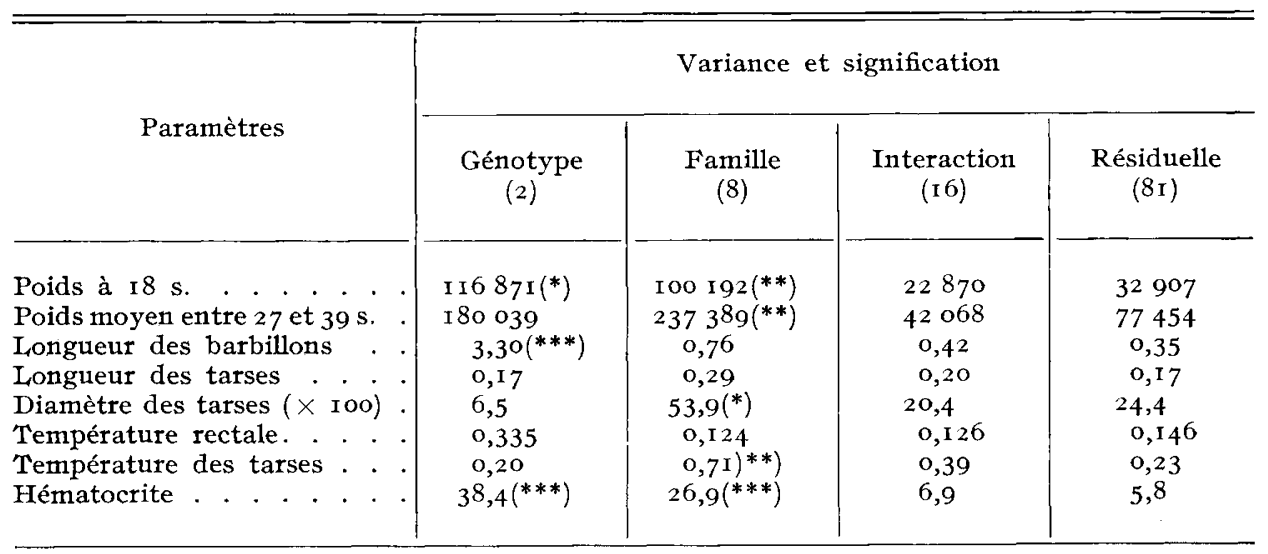

- Entre parenthèses ( ) : d.1.

- $\left(^{*}\right)\left({ }^{*}\right)\left({ }^{* *}\right)$ : significatif respectivement au seuil 5 , I et $0, \mathrm{I}$ p. roo. 
TABLEAU 5

Moyennes par lots, tous génotypes réunis

Mean values according to treatment, genotypes being grouped

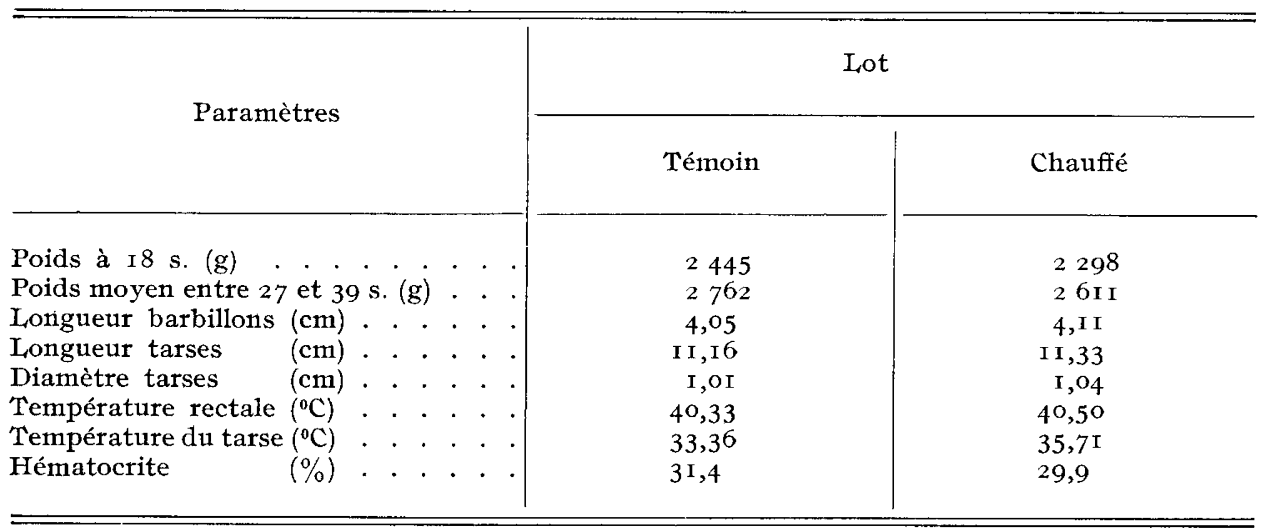

TABLEAU 6

Récapitulation des significations des analyses de variance à 3 voies

Significances of 3-way variance analyses

\begin{tabular}{|c|c|c|c|c|c|c|}
\hline \multirow{2}{*}{ Paramètres } & \multicolumn{3}{|c|}{ Effets principaux } & \multicolumn{3}{|c|}{ Interactions } \\
\hline & $\begin{array}{l}\text { (I) }= \\
\text { Lot }\end{array}$ & $\begin{array}{c}(2)= \\
\text { Génotype }\end{array}$ & $\begin{array}{c}(3)= \\
\text { Famille }\end{array}$ & $(\mathrm{I}) \times(2)$ & $(\mathrm{I}) \times(3)$ & $(2) \times(3)$ \\
\hline $\begin{array}{l}\text { Poids à I } 8 \mathrm{~s} . \\
\text { Poids moyen entre } 27 \text { et } \\
39 \text { s. . . . . . } \\
\text { Longueur barbillons } \\
\text { Longueur tarses } \\
\text { Diamètre tarses } \\
\text { Température rectale. } \\
\text { Température tarse . } \\
\text { Hématocrite }\end{array}$ & $\begin{array}{l}(* * *) \\
(* * *) \\
(* * *) \\
(* *) \\
(* *) \\
(* *) \\
(* *)\end{array}$ & $\begin{array}{c}(* * *) \\
(* *) \\
(* *) \\
(* * *)\end{array}$ & $\begin{array}{l}(* * *) \\
(* * *) \\
(* * *) \\
(* * *) \\
(* * *) \\
(* *) \\
(* * *)\end{array}$ & $\begin{array}{l}(* * *) \\
(* * *)\end{array}$ & $\begin{array}{c}(* *) \\
(*) \\
(*) \\
(* * *)\end{array}$ & \\
\hline
\end{tabular}

$(*)\left({ }^{* *}\right)\left(*^{* *}\right)$ : variance significative respectivement au seuil de 5 , r et o, I p. Ioo.

En lot chauffé, (tab1. 3 et 4 ), le poids adulte est atteint plus tardivement; au-delà de 27 semaines, l'accroissement du poids corporel est encore sensible, (environ $30 \mathrm{~g}$ par 28 jours de 3 I à 39 semaines). La différence de poids vif entre génotypes diminue en valeur relative avec 1'âge : à ro semaines, $N a N a$ surclasse $n a^{+} n a^{+}$et $N_{n} a^{+}$respectivement de II et 3 p. Ioo; à I 8 semaines, de 6,5 et 2 p. Ioo; de 5 et I,4 p. Ioo à 27 semaines. Ces différences s'accentuent légèrement 


\section{TABLEAU 7}

Valeurs moyennes par génotype des variables mesurées seulement en lot chauffè Mean values per genotype: variables measured only in the heated group

\begin{tabular}{|c|c|c|c|}
\hline \multirow{2}{*}{ Paramètres } & \multicolumn{3}{|c|}{ Génotype } \\
\hline & $\mathrm{NaNa}$ & $\mathrm{Nana}^{+}$ & $n a+n a^{+}$ \\
\hline 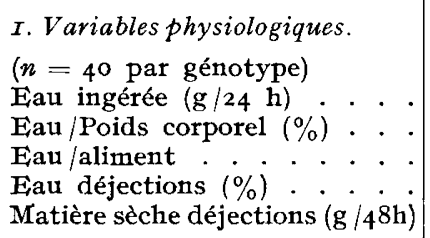 & $\begin{array}{c}422,6 \\
15,62 \\
3,19^{a} \\
80,8 \\
96,3^{a}\end{array}$ & $\begin{array}{c}421,6 \\
15,92 \\
3,33^{a} \\
80,0 \\
72,6^{a b}\end{array}$ & $\begin{array}{c}459,4 \\
18,48 \\
3,87^{b} \\
80,4 \\
61,4^{b}\end{array}$ \\
\hline $\begin{array}{l}\text { 2. Variables anatomiques. } \\
(n=\text { I I par génotype) } \\
\text { Poids plumes p. Ioo }\left({ }^{1}\right) \\
\text { Graisse abdominale p. Ioo }\left({ }^{2}\right) \\
\text { Thyroïdes }\left(p \text {. roo } \times 10^{5}\right)\left({ }^{2}\right) \\
\text { Surrénales }\left(p . \text { Ioo } \times 1^{5}\right)^{5}\left(^{2}\right)\end{array}$ & $\begin{array}{l}3,5 \mathrm{I}^{a} \\
5,66^{a} \\
\mathrm{I}, 28^{a} \\
0,5^{a}\end{array}$ & $\begin{array}{l}4,37^{b} \\
5,34^{a} \\
1,09^{a b} \\
0,57^{a}\end{array}$ & $\begin{array}{l}6,08^{c} \\
3,7^{b} \\
1,01^{b} \\
0,69^{b}\end{array}$ \\
\hline
\end{tabular}

Les moyennes suivies de lettres différentes sont significativement différentes.

(1) p. Ioo du poids saigné.

(2) p. roo du poids plumé.

\section{TABLEAU 8}

Variables mesurées en lot chauffé. Analyses de variance

Variables measured only in the heated group: variance analysés

\begin{tabular}{|c|c|c|c|c|}
\hline \multirow{2}{*}{ Paramètres } & \multicolumn{4}{|c|}{ Variance et signification } \\
\hline & $\begin{array}{c}\text { Génotype } \\
\text { (2) }\end{array}$ & $\begin{array}{c}\text { Famille } \\
(8)\end{array}$ & $\begin{array}{l}\text { Interaction } \\
\text { (I6) }\end{array}$ & $\begin{array}{l}\text { Résiduelle } \\
\quad(79)\end{array}$ \\
\hline Consommation d'eau/jour & I 5 I95 & IO 210 & 7877 & 16803 \\
\hline Eau/poids corporel (p. Ioo) & 70,68 & 24,53 & II, 50 & 27,54 \\
\hline Eau/aliment.... & $4,19\left(^{*}\right)$ & $\mathrm{I}, \mathrm{O} 2$ & 0,48 & $\mathrm{I}, 02$ \\
\hline P. Ioo eau déjections. . & 7,49 & $226,33(* * *)$ & $4 \mathrm{I}, 49$ & 51,69 \\
\hline Mat. sèches déjections (g) & $3462\left({ }^{*}\right)$ & $3 \mathrm{I} 74\left(^{(* *}\right)$ & I 103 & 979 \\
\hline
\end{tabular}

$\left(^{*}\right)\left({ }^{* *}\right)\left({ }^{* *}\right)$ : significatif respectivement au seuil 5 , I et $0, \mathrm{I}$ p. Ioo; entre parenthèses ( ) : d.1. 
après 3 I semaines et atteignent en fin d'expérience 7 et 2 p. Ioo. En valeur absolue, la variation de poids entre $\mathrm{I} 8$ et 39 semaines est à petu près équivalente entre génotypes.

Le tableau 6 synthétise ces résultats en montrant en particulier l'existence d'une interaction très hautement significative entre lot et génotype pour le poids corporel aux divers âges. A âge égal, chez 1'individu normalement emplumé $\left(n a^{+} n a^{+}\right)$, le poids vif en lot chauffé est très nettement inférieur à celui du lot témoin et ceci jusqu'au stade adulte. Ceci est conforme aux observations de nombreux auteurs (par exemple WILson, I949; Huston et al., I957; Campos et al., I960; MUELleR, I96r; PAYNe, I965, I966; ITo et al., I97I, I972; DAvis et al., I972; Chima, I975; El JACK et Blum, I978; Petersen et Horst, I978). En ce qui concerne les homozygotes mutants, jusqu'à I8 semaines la croissance moyenne est plus rapide en lot témoin, puis elle devient meilleure en lot chauffé. Ainsi, les poulettes ou pondeuses de type sauvage occupent les positions extrêmes dans chaque lot : meilleure croissance en lot témoin, moins bonne en lot chauffé. L'hétérozygote est partout intermédiaire.

\section{2. - Paramètres morphologiques, températures corporelles et hématocrite}

L'effet du génotype sur la longueur des tarses est faible et significatif seulement en lot témoin, où il va dans le même sens que l'effet sur les poids corporels. Dans l'ensemble, les températures élevées tendent à augmenter cette variable, en sens contraire de l'effet sur la croissance pondérale. Il n'y a pas d'interaction significative lot $\times$ génotype.

Le diamètre des tarses est relativement peu variable. Aucun effe $t$ significatif associé au génotype n'apparaît pour ce caractère.

Par contre, la longueur des barbillons est significativement plus grande chez l'individu $N a N a$ comparé à l'individu sauvage $n a^{+} n a^{+}$, en lot témoin comme en lot chauffé, alors qu'il n'y a pas de différences entre lots pour ce critère. Cet effet associé au gène "cou nu " va dans le même sens que celui observé sur coquelets à Io semaines (MonNe't et al., I979).

La température rectale, en lot témoin, est significativement plus faible, d'environ 2/Io de degré, chez l'homozygote $N a N a$ comparé à l'homozygote sauvage ou à l'hétérozygote. Les résultats obtenus pour la température de la patte sont de même sens. En lot chauffé, les températures rectales augmentent en moyenne de I/ Io de degré quand on passe du génotype $\mathrm{NaNa}$ à $\mathrm{Nana}^{+}$et de $N a n a^{+}$à $n a^{+} n a^{+}$, mais ces écarts ne sont pas significatifs. Dans ce lot, la température de la patte ne présente pas de variation appréciable associée au locus $N a$. Du tableau 6 ressort un effet global de la température d'élevage, la chaleur augmentant légèrement les températures corporelles, et, un effet global du génotype au locus $N a$. La température de la patte suit la même tendance, mais, en lot chauffé, outre sa valeur supérieure en moyenne, ses variations sont plus réduites du fait de la température ambiante élevée. Le résultat obtenu en lot chauffé pour la température rectale est analogue à celui obtenu sur jeunes coquelets (MONNET et al., I979), et va dans le sens d'une meilleure tolérance à la chaleur liée au gène "Cou nu ".

Le taux d'hématocrite est équivalent pour $\mathrm{NaNa}, \mathrm{Nana}^{+}$et $n a^{+} n a^{+}$en lot témoin. En lot chauffé, il apparaît plus élevé, de façon hautement significative, chez les individus porteurs du gène $N a$. Le tableau 6 montre l'absence d'effet significatif associé, dans l'ensemble, au gène $N a$, et une interaction (significative 
au seuil 5 p. Ioo) entre gène "Cou nu " et température. Ce même tableau et le tableau 5 indiquent d'autre part une diminution globale du taux d'hématocrite en ambiance chaude. Ceci rejoint les conclusions d'autres auteurs (ZIMMERMAN et al., I972, I973; ANDRADE et al., 1976, I977).

\section{3. - Variables liées à l'ingestion et à l'excrétion d'eau en lot chauffé. (Tab1. 7 et 8)}

Les différences entre génotypes relatives à la consommation journalière d'eau ne sont pas significatives : toutefois, cette consommation est légèrement plus élevée chez $n a^{+} n a^{+}$en valeur absolue. La différence est plus nette lorsque la consommation d'eau est ramenée au poids corporel ou à la consommation journalière d'aliment; dans ce dernier cas, elle est significativement supérieure pour le génotype $n a^{+} n a^{+}$.

Chez les pondeuses de type sauvage, la quantité d'eau et de matières sèches des déjections est plus faible en valeur absolue que pour les pondeuses $\mathrm{NaNa}$ ou $\mathrm{Nana}^{+}$, en corrélation avec la quantité respective d'aliment ingéré. Les différences dans le pourcentage d'eau des fientes sont très faibles. En rapprochant les résultats qui précèdent, on s'aperçoit que $n a^{+} n a^{+}$consomme en moyenne 8 p. Ioo d'eau en plus et en rejette I9 p. Ioo en moins dans les déjections que $N a N a$.

\section{4. - Paramètres anatomiques en lot chauffé}

a) La quantité de plumes ramenée au poids corporel saigné (ainsi d'ailleurs qu'en valeur absolue) est très significativement différente entre les trois génotypes, dans l'ordre décroissant $n a^{+} n a^{+}, \mathrm{Nana}^{+}, \mathrm{NaNa}$.

b) Pour la graisse abdominale, des différences hautement significatives sont liées au gène " cou nu », en valeur absolue et en pourcentage du poids plumé.

c) Poids des thyroides et des surrénales: La différence de poids des thyroïdes entre les génotypes homozygotes est significative en valeur absolue et en p. Ioo; l'individu sauvage a des thyroïdes plus petites, l'écart étant voisin de $24 \mathrm{p}$. Ioo. L'hétérozygote est intermédiaire. Pour le poids des surrénales, l'individu $n a^{+} n a^{+}$ est significativement supérieur aux deux génotypes porteurs du gène "Cou nu ".

Nos résultats concernant la taille des thyroïdes concordent avec celui obtenu par BoRDAs et al. (I978) sur coquelets à température élevée.

\section{Discussion et conclusions}

En ce qui concerne la quantité de plumage, la dominance incomplète du gène "Cou nu " a été observée qualitativement par CRAWFORD (I976), et ScoT'T et CRAWFORD (I977). Nous l'avons constatée pour le poids total du plumage rapporté au poids corporel chez le jeune des deux sexes à Io semaines d'âge (BoRDAs et al., I978; MONNET et al., I979). Elle se manifeste encore très nettement ici, chez la poule adulte, en lot chauffé. En valeur relative, la réduction du plumage associée au gène $N a$ à ce stade est du même ordre qu'au stade juvénile. Indépendamment du génotype, si l'on compare les poids relatifs du plumage sur individus jeunes 
(MON NE'T et al., I979) et sur poules adultes (tabl. 7 du présent article), on voit qu'ils sont inférieurs dans le second cas. Cette réduction peut s'expliquer si la quantité de plumes est, au moins approximativement, proportionnelle à la surtace corporelle évaluée par la quantité $a \mathrm{P}^{2 / 3}, \mathrm{P}$ représentant le poids; lorsque ce dernier augmente, le rapport $\frac{a \mathrm{P}^{2 / 3}}{\mathrm{P}}$ décroît.

Mobnet et al. (I979) ont observé que le gène "Cou nu " a un effet favorable sur la croissance jusqu'à l'âge de ro semaines dans les deux sexes en ambiance chauffée. Des observations ultérieures (Bordas et al., r980) montrent de même chez des pondeuses placées en ambiance chaude par comparaison avec une ambiance tempérée, un ensemble d'effets favorables associés au gène $N a$, en particulier sur la consommation alimentaire, le poids moyen des oufs et celui de leurs composants.

Les résultats présentés ici montrent que 1'homozygote "Cou nu " s'accommode moins bien que les autres génotypes des températures relativement basses appliquées en lot témoin, surtout dans la période allant de ro à 27 semaines d'âge. Au contraire, ces mêmes résultats, comme ceux cités précédemment, vont dans le sens d'une meilleure adaptation à une chaleur constante des poulettes ou poules adultes à plumage "Cou nu " (homozygotes et à un moindre degré hétérozygotes). Ceci se traduit en premier lieu par les effets sur la croissance entre ro semaines et 1'âge adulte et sur la lipogenèse adulte.

Ces résultats paraissent attribuables à l'effet du gène $N a$ sur le plumage et par là sur la thermolyse, effet persistant tout au long de la vie. La réduction pondérale de plumage reflète une augmentation des surfaces non emplumées, d'où un accroissement des pertes de chaleur sensible par rayonnement ou convexion. Notons qu'en plus de l'effet sur le plumage, l'augmentation de la taille des barbillons (et corrélativement de la crête) apportée par le gène $N a$ peut aussi constituer un élément favorable en ambiance chaude, si 1'on se réfère au rôle relativement important de ces organes non emplumés dans les processus de thermorégulation (STUR KIE, I976).

En compensation de cette isolation thermique inférieure, on s'attend à ce que l'ingéré énergétique soit plus important. C'est ce que confirment les données sur la consommation alimentaire présentées dans un article séparé (BORDAS et al., I980). D'autre part, dans la mesure où la différence de taille des thyroïdes entre génotypes, évaluée dans le lot chauffé, reflète aussi une différence d'activité de ces glandes, sa relation causale avec cette thermogenèse accrue chez les animaux porteurs du gène "Cou nu ", paraît vraisemblable. FREEMAN (I97I) rappelle le rôle important de ces glandes à cet égard, dès l'éclosion. La légère baisse de la température rectale et celle des extrémités chez les poules "Cou nu " semble d'ailleurs indiquer que l'augmentation de thermogenèse réalisée ne compense pas tout à fait celle de la thermolyse.

Cette thermolyse plus élevée en présence du gène $N a$ suggère une explication des différences observées pour d'autres paramètres. La plus grande ingestion d'eau des poules à plumage normal comparées aux poules "Cou nu ", relativement à leur consommation d'aliment, et le fait qu'au contraire on retrouve moins d'eau dans leurs déjections, peut difficilement correspondre à une rétention d'eau supérieure ou à une plus grande exportation dans les oufs. En effet, la consommation alimentaire et le poids corporel de ce génotype sont les plus faibles et leur production d'œufs n'est pas supérieure à celle des autres génotypes. L'hypothèse la plus plausible est celle d'une évaporation pulmonaire plus intense de cette eau par les 
poules $n a^{+} n a^{+}$pour contribuer à la régulation thermique : le rôle de l'évaporation chez les poules en stress thermique est bien connu.

Quant à la graisse abdominale, nos résultats semblent concorder avec ceux de TOUChBuRn et BLUm (I972) et Bordas et al. (I978) pour le stade juvénile. Les premiers auteurs concluent que la présence du gène " Cou nu " détermine une augmentation de la lipogenèse. Les données présentes suggèrent la même conclusion, sans que l'on puisse faire la part de ce qui revient simplement à l'ingestion accrue d'aliment causée par le gène $N a$.

Enfin, la taille plus faible des surrénales chez les poules "Cou nu " à température élevée peut être un autre indice d'une meilleure adaptation physiologique, dans la mesure où elle représente une activité moindre de ces glandes. On peut penser que le "stress" subi pour les animaux "Cou nu " du fait de la chaleur est moins important; or FREEMAN (I976), par exemple, mentionne 1'hypertrophie des surrénales comme 1'un des signes possibles de "stress" chez les volailles. Cette hypothèse nécessiterait toutefois, bien entendu, d'être vérifiée plus directement.

Reçu pour publication en Novembre 1980.

\section{Summary}

\section{Naked neck gene, body weight and anatomical and physiological traits of pullets and adult hens according to ambient temperature}

Females of three genotypes $\mathrm{NaNa}$ (naked neck homozygotes), $\mathrm{Nana}^{+}$(naked neck heterozygotes) and $n a+n a^{+}$(normal plumage), hatched from the same parents, were distributed in two treatment groups : control (kept at moderate temperature with fluctuations according to climatic variations) and heated (constant $3 \mathrm{r}^{\circ} \mathrm{C}$ from 4 to 39 weeks of age). In the control group, average weights of the three genotypes differed little at ro weeks of age, but after that age the body weight gain was considerably inferior for the $N a N a$ genotype compared to $n a^{+} n a^{+}$, and consequently body weights at 18,27 and 39 weeks of age were lower for naked neck homozygotes, and accordingly their mean shank length was slightly inferior. On the contrary, wattle length was superior for $N a N a$ females. Their rectal temperature and surface temperature of their shanks were slightly lower than for hens with normal plumage. The heterozygous genotype was generally intermediate, but closer to the $N a N a$ homozygote for wattle and shank length and shank temperature, and closer to the $n a^{+} n a^{+}$genotype for rectal temperature.

In the heated group, at io and at 18 weeks of age, homozygous naked neck birds are the heaviest and heterozygotes are intermediate, but the differences decrease with age and are no more significant from 27 weeks. As in the control group, naked neck hens have larger wattles. Their rectal and superficial temperature is also slightly inferior, but here the difference is not significant. At high temperature hens with normal plumage have a lower hematocrit value.

Body weight at I 8 weeks, adult body weight, shank temperature and hematocrit show a significant genotype $\times$ environment interaction.

Several physiological or anatomical variables were measured only in the heated group. The ratio of water intake to food intake is lower for naked neck than for normal hens. Dry matter in feces for them is higher, which is consistent with their higher food intake. Weight of plumage in per cent of body weight increases from the $\mathrm{NaNa}$ genotype to $\mathrm{Nana}^{+}$and to $n a^{+} n a^{+}$ which has the highest value. This corresponds to the result formerly found at io weeks of age. Naked neck hens have more abdominal fat, larger thyroids and smaller adrenals.

\section{Remerciements}

Nous remercions le $\mathrm{D}^{\mathrm{r}} \mathrm{P}$. Mongin et F. H. RICARD, Station de Recherches avicoles, 37-Nouzilly, pour leurs utiles remarques concernant ce manuscrit. 


\section{Références bibliographiques}

DE ANDrade A. N., ROgLER J. C., FEatherston W. R., I976. Influence of constant elevated temperature and diet on egg production and shell quality. Poult. Sci., 55, 685-693.

De ANdrade A. N., Rogler J. C., Featherston W. R., Alitiston C. W., i977. Interrelationships between diet and elevated temperatures (cyclic and constant) on egg production and shell quality. Poult. Sci., 56, I I 78 -r I 88.

Bordas A., Múrad P., Sergent D., Ricard F. H., I978. Influence du gène Na (" cou nu ") sur la croissance, la consommation alimentaire et la composition corporelle du poulet selon la température ambiante. Ann. Génét. Sél. Anim., 10, 209-23r.

BORDAS A., MONNET L. E., MÉ́RAT P., I980. Gène cou $n u$, performances de ponte et efficacité alimentaire selon la température chez la poule. Ann. Génét. Sél. Anim., 12 (sous presse).

Campos A. C., Wilcox F. H., Shaffner C. S., ig6o. The influence of fast and slow changes in ambiant temperature on production traits and mortality of laying hens. Poult. Sci., 39, II9-I 29.

Снiма M. M., 1975. Untersuchungen zum Einfuss hoher Dauertemperaturen auf Körpertemperatur, gewichtsentwicklung und Leistungsreaktion von leiehten und mittelschweren Legehybriden. Thèse, Berlin.

CRAWFORD R. D.,.I976. Incomplete dominance of the gene for Naked Neck in the domestic fowl. Poult. Sci., 55, 820-822.

Davis R. H., Hassan O. E., Sykes A. H., I972. The adaptation of energy utilization in the laying hen to warm and cool ambient temperature. J. Agric. Scci., 79, 363-369.

EI, JACK M. M., BLUM J. C., I978. The influence of high constant environmental temperature and energy level in the diet on the performance of the laying hen. Arch. Geflügelk., 42, 2 I6-220.

FREEMAN B. M., I97I. Body temperature and thermoregulation. In Physiology and Biochemistry of the Domestic fowl. Ed. BELL D. F., FrEEMAN B. M., Academic Press, London.

FREEMAN B. M., I976. Stress and the domestic fowl: a physiological re-appraisal. World's Poult. Sci. J., 32, 249-256.

Huston T. M., Jolner W. P., Carmon J. L., I957. Breed differences in egg production of domestic fowl at high environmental temperature. Poult. Sci., 36, I247-1 254.

HutT F. B., I949. Genetics of the fowl. McGraw Hill, New-York.

ITO T., Moriya T., Yamamoto S., MrmuRa K., r97I. Effects of environmental temperature on egg production, food intake and water consumption in laying White Leghorns. J. Fac. Fisk. Anim. Husb. Hiroshima Univ., 9, I5I-I6o.

ITo T., Ozaka T., Shishido H., Mimura K., r972. Effects of environmental temperature on egg production, food intake and water consumption in laying White Leghorns. III. Effects of humidity. J. Fac. Fish. Anim. Husb. Hiroshima Univ., 11, 23-31.

Monnet I. E., Bordas A., MERAT P., I979. Gène Cou nu et performances de croissance selon la température chez le poulet. Ann. Génét. Sél. Anim., 11, 397-412.

MUELLER W. J., I96r. The effect of constant and fluctuating environmental temperatures on the biological performance of laying pullets. Poult. Sci., 40, I562-I571.

PAyNe C. G., 1965. The influence of environmental temperature on poultry performance. Proc. $2 d$ Europ. Poultry Cont. Bologna, I964, I I-I2o.

PAYNe D. G., I966. Environmental temperature and the performance of light breed pullets. Proc. I3th Wld's Poult. Congress, Kiev, 480-485.

Petersen J., HorsT P., x978. Hohe Umwelttemperaturen als auslösender Faktor von GenotypUmwelt Interaktionen beim Legehuhn. Arch. Geflügelk., 42, I73-I78.

RICARD F. H., r974. Étude de la variabilité génétique de quelques caractéristiques de carcasse en vue de sélectionner un poulet de qualité. C. R. I er Congrès mondial de Génétique appliquée à la production animale, Madrid, 1, 93I-940.

Ricard F. H., Rouvier R., I967. Étude de la composition anatomique du poulet de chair. I. Variabilité de la répartition des différentes parties corporelles chez des coquelets Bresse Pile. Ann. Zootech., 16, 23-39.

SCOTT T., CRAwFORd R. D., I977. Feather number and distribution in the throat tuft of naked neck chicks. Poult. Sci., 56, 686-688.

SMITH T., LEE R., 1973. A Study of the Naked neck gene of the fowl. Poult. Sci., 56, I 758 (abstr.).

StUrkit P. D., 1976. Avian Physiology. $3^{\mathrm{e}}$ ed. Springer, New-York. 
Touchburn S. P., Blum J. C., I972. Effects of the gentes for dwarfism (dw) and naked neck $(\mathrm{Na})$ on chick growth and lipid metabolism. Ann. Génét. Sél. Anim., 4, 3I I-3I6.

WILSON W. O., I949. High environmental temperature as affecting the reaction of laying hens to iodinated casein. Poult. Sci., 28, 581-592.

Zimmerman R. A., SNetringer D. C., GReEne D. E., MarR J. E., 1972. Effect of environmental stress on layer performance and egg characteristics. Poult. Sci., 51, r889 (abstr.).

Zimmerman R A., Snetsinger D. C., GreEne D. E., I973. Significance of day length and air circulation for commercial layers under heat stress. Poult. Sci., 52, 2106 (abstr.). 